\title{
Three Different Strains of Heat-Killed Group B $\beta$-Hemolytic Streptococcus Cause Different Pulmonary and Systemic Hemodynamic Responses in Conscious Neonatal Lambs
}

\author{
ROBERT F. COVERT AND MICHAEL D. SCHREIBER \\ Department of Pediatrics, Sections of Neonatology [R.F.C., M.D.S.J and Critical Care Medicine [M.D.S.J. Wyler \\ Children's Hospital. Pritzker School of Medicine, Biological Sciences Division. University of Chicago, Illinois \\ 60637
}

\begin{abstract}
Although group B $\beta$-hemolytic streptococcus (GBS) causes pathologic hemodynamic alterations in both human neonates and neonatal animal models of sepsis, little is known about strain-dependent differences in hemodynamic responses to GBS. This study compared pulmonary and systemic hemodynamic dose-response profiles in conscious neonatal lambs with three different strains of heat-killed GBS originally isolated from infected human neonates (group 1: serotype Ib, early-onset sepsis; group 2: serotype Ib, necrotizing enterocolitis; and group 3: serotype III, meningitis). Regression models of hemodynamic responses were characterized after lambs were injected with heat-killed GBS (dose range 0.1-6.0 × 109 colonyforming units, i.v.). All three GBS strains caused dosedependent increases in mean pulmonary arterial pressure and pulmonary and systemic vascular resistances and decreases in cardiac output and heart rate. The GBS strain used in group 1 caused a greater effect on mean pulmonary arterial pressure and systemic vascular resistance than those used in groups 2 and 3 and was the only strain to cause an increase in mean systemic arterial pressure. The GBS strains used in groups 1 and 2 had a greater effect on pulmonary vascular resistance than that used in group 3 . No group differences were observed in cardiac output and heart rate responses, which were, however, influenced by age, gender, and duration of postoperative recovery of the lambs. No attenuation or augmentation of hemodynamic effect was observed after sequential doses of $10^{9}$ colonyforming units of GBS given in a single day. This study demonstrates strain-dependent quantitative differences in pulmonary vascular response and qualitative differences in systemic vascular response to heat-killed GBS. (Pediatr Res 33: 373-379, 1993)
\end{abstract}

\section{Abbreviations}

GBS, group B $\beta$-hemolytic streptococcus cfu, colony-forming unit

$P_{\mathrm{pa}}$, mean pulmonary arterial pressure

$R_{p}$, pulmonary vascular resistance

$P_{\mathrm{sa}}$, mean systemic arterial pressure

$R_{s}$, systemic vascular resistance

$\mathrm{Q}_{\mathrm{pa}}$, cardiac output

Received July 9, 1992; accepted November 18, 1992

Correspondence and reprint requests: Robert F. Covert, M.D., Department of Pediatrics. University of Chicago, MC 6060. 5841 South Maryland Ave., Chicago. IL 60637 .

Portions of this research were supported by a grant from the American Lung Association (M.D.S.). This research was performed at the University of Chicago and at the Michael Reese Hospital and Medical Center. Chicago, IL.
HR, heart rate

$\mathrm{PaO}_{2}$, arterial oxygen tension

$\mathrm{PaCO}_{2}$, arterial carbon dioxide tension

$\mathrm{SaO}_{2}$, arterial oxyhemoglobin saturation

ANOVA, analysis of variance

GBS is the leading cause of bacterial pneumonia and sepsis in the human neonate and remains an important cause of shock, respiratory failure, persistent pulmonary hypertension, and death in the neonatal period $(1-7)$.

Infusion into animals of live GBS bacteria isolated from infected human neonates produces cardiac depression (8-12), splanchnic hypoperfusion $(12,13)$, and pulmonary hypertension $(8-22)$ in several neonatal animal models. In addition, a pulmonary pressor response to infusion of heat-killed GBS has been described in conscious piglets (23), in conscious yearling sheep (14), and in neonatal lambs $(24,25)$. Although previous studies have used various serotypes of GBS, the similarities or differences between different GBS strains on pulmonary and systemic hemodynamic responses in animal models have not been adequately studied.

Thus, we designed this study to compare and contrast pulmonary and systemic hemodynamic dose-response profiles in conscious neonatal lambs with three different GBS strains, to evaluate other experimental conditions that may influence the hemodynamic responses to GBS, and to evaluate whether sequential doses of heat-killed GBS in a single day would lead to attenuation or augmentation of hemodynamic effects.

\section{MATERIALS AND METHODS}

Animal care and procedures were approved by the Institutional Animal Care and Use Committee of Michael Reese Hospital and Medical Center.

Chronic Surgical Preparation. Thirteen mixed-breed newborn lambs (eight female) underwent instrumentation at age $1-16 \mathrm{~d}$ (mean $5 \pm 1 \mathrm{~d}$ ) while anesthetized with $0.5-1.5 \%$ isoflurane delivered by a small-animal ventilator; the details of instrumentation and recovery have been previously described (24-26).

Briefly, polyvinyl vascular catheters were advanced into the descending aorta and inferior vena cava through vessels exposed by an incision in the lower hindlimb. A left-sided thoracotomy and pericardiotomy were performed to ligate the ductus arteriosus and to place a blood flow transducer around the main pulmonary artery. In the first nine lambs, this transducer was electromagnetic (Carolina Medical Electronics, King, NC); in 
four later lambs, this transducer was ultrasonic (Transonics Systems, Inc., Ithaca, NY). Baseline values for pulmonary blood flow volume for these two measurement systems were not different in the lambs. In addition, vascular catheters were placed into the internal mammary vein (advanced into the right atrium) and artery, the pulmonary artery, and the left atrium for the measurement of vascular pressures and arterial blood $\mathrm{pH}$ and gas tensions. The flow transducer cable and catheters were exteriorized and secured to the lamb's flank. After recovery, lambs were returned to their ewes to recover for several days before study. Lambs were given procaine penicillin G 400000 units, streptomycin $500 \mathrm{mg}$, and gentamicin $10 \mathrm{mg}$ intramuscularly daily for $5 \mathrm{~d}$ beginning the day of surgery and were also given the penicillin and streptomycin after any GBS study. Vascular catheters were cleared and refilled with heparin regularly. Satisfactory catheter position was confirmed at postmortem examination when studies were complete.

Measurements and Calculations. Vascular pressures, including pulmonary and systemic arterial and left and right atrial, were measured with Gould-Statham P23ID transducers (Gould Inc. Cleveland, $\mathrm{OH}$ ). Puimonary artery blood flow was measured with Carolina FM501 flowmeters or Transonics T201 flowmeters as appropriate. A Gould 3800 direct-writing pen recorder was interfaced with these instruments and continuously recorded vascular pressures and pulmonary blood flow. HR was determined from the phasic arterial tracing. Vascular pressure transducers and amplifiers were calibrated before each study and flow transducers were precalibrated before implantation. Zero references for physiologic variables were checked regularly throughout a study. Arterial blood gas tensions and pH (Radiometer ABL30. Copenhagen. Denmark) and Hb concentration and oxygen saturation (Radiometer OSM-3) were measured at points defined in the protocol, after being drawn from the descending aorta. Blood gas tensions and $\mathrm{pH}$ were corrected to $39.5^{\circ} \mathrm{C}$, the lambs' estimated temperature.

$\mathrm{Q}_{\mathrm{pa}}$ was measured as pulmonary blood flow and indexed to animal weight and reported as $\mathrm{mL} \cdot \mathrm{min}^{-1} \cdot{ }^{\mathrm{kg}-1}$. Peak $R_{p}$ and $R$, were calculated with peak $P_{p a}$ and $P_{s a}$ and simultaneous atrial pressures and $\mathrm{Q}_{\mathrm{pa}}$ as follows: $\mathrm{R}_{\mathrm{p}}=\left(\mathrm{P}_{\mathrm{pa}}-\mathrm{P}_{\mathrm{la}}\right) / \mathrm{Q}_{\mathrm{pa}}$ and $\mathrm{R}_{\mathrm{s}}=\left(\mathrm{P}_{\mathrm{sa}}\right.$ $\left.-P_{r a}\right) / Q_{p a}$, where $P_{l a}$ and $P_{r a}$ are mean left atrial pressure and mean right atrial pressure, respectively. Stroke volume was calculated with peak $\mathrm{Q}_{\mathrm{pa}}$ and simultaneous $\mathrm{HR}$ in $\mathrm{mL} \cdot \mathrm{kg}^{-1} \cdot$ beat $^{-1}$ as $\mathrm{Q}_{\mathrm{pa}} / \mathrm{HR}$.

GBS Clinical Syndromes and Serotypes. Each of the three different strains of GBS used in this study was isolated from the blood of an infected human neonate. Lambs treated in group 1 were infused with GBS serotype Ib isolated from a very-lowbirth-weight preterm neonate with fatal early-onset sepsis, pneumonia, shock, meningitis, and severe hypoxemia; this strain has been the standard GBS used in previous studies from our laboratory $(24,25)$ and in previous studies of our colleagues $(10-13$. 20). Lambs treated in group 2 were infused with a different GBS serotype Ib isolated from a 32-wk gestation preterm neonate during an episode of necrotizing enterocolitis in the 3 rd week of life that was associated with neutropenia but not pneumonia, meningitis, or cardiovascular collapse. Lambs treated in group 3 were infused with GBS serotype III, previously designated M732 (strain 31475, ATCC catalogue, Rockville, MD) isolated from an infant with meningitis.

Analysis of serotypes of all three strains of GBS was performed by immunofluorescence with rabbit antisera in the laboratory of Kenneth M. Boyer, M.D., at Rush-Presbyterian-St. Luke's Medical Center in Chicago, IL.

GBS Preparation. Live GBS strains were prepared by previously described techniques (10-13). In summary, GBS strains were grown in $250 \mathrm{~mL}$ of Todd-Hewitt broth to late-log phase $\left(10^{9} \mathrm{cfu} \cdot \mathrm{mL}^{-1}\right)$. The bacteria were then centrifuged, the supernatant decanted, and the organisms resuspended to their original concentration in sterile normal saline.

In addition, bacteria were then killed by heating the suspension to $60^{\circ} \mathrm{C}$ for $60 \mathrm{~min}$ as previously described $(24,25)$; death of the bacteria was subsequently verified by failure to isolate bacterial growth from a sample of the heated suspension on blood agar. Suspended, heat-killed GBS was stored in aliquots at $-70^{\circ} \mathrm{C}$ until the study day. Growth and preparation of each of the three different strains of GBS, from recovery to killing to storage, were identical.

Experimental Protocols. Lambs were studied while awake, breathing room air, resting quietly upright in a sling, and attached appropriately to all instrumentation.

Protocol 1 . At the time of study, lambs in this protocol were 4-35 d (mean 14 $\pm 2 \mathrm{~d}$ ) of age and had recovered 3-27 d (mean $9 \pm 2$ d) from thoracotomy; gender, postnatal age at the time of study, and duration of recovery from thoracotomy were similar between the three treatment groups.

Lambs in each of the three treatment groups received i.v. bolus injections of varying amounts of heat-killed GBS in random order in a single day; the possible doses included $0.10,0.25,0.50$, $1.0,3.0$, and $6.0 \times 10^{9} \mathrm{cfu}$ (maximum volume $6 \mathrm{~mL}, n=80$ total doses). Injections were given over $<10 \mathrm{~s}$. Twelve lambs were treated in group 1 (serotype $\mathrm{Ib}$, carly-onset sepsis) and received from one to six injections a day ( $n=32$ total doses). Four lambs were treated in group 2 (serotype Ib, sepsis with necrotizing enterocolitis) and each received six injections per day $(n=24$ doses). Four lambs were treated in group 3 (serotype III, meningitis) and also received six injections per day ( $n=24$ doses). Inclusion of an individual lamb into more than one treatment group was infrequent; no single lamb accounted for more than seven of the 80 total injections.

Baseline values consisted of hemodynamic measurements and arterial blood gas samples obtained during quiet rest and after a stabilization period of hemodynamic measurements. After each injection of GBS, hemodynamic measurements were continuously monitored and arterial blood gas samples were obtained at 5 and $30 \mathrm{~min}$ after injection. Peak responses of measured $\mathrm{P}_{\mathrm{pa}}$, $P_{\text {sa }}$, mean left atrial pressure, mean right atrial pressure, and $Q_{p a}$ were assessed from the continuous recording; peak responses of calculated $R_{p}, R_{s}$, and stroke volume were determined as previously described. HR was assessed at $5 \mathrm{~min}$ after injection. Hemodynamic responses were permitted to stabilize for at least $30 \mathrm{~min}$ before a subsequent injection.

Protocol 2. This protocol was conducted specifically to examine whether hemodynamic responses to heat-killed GBS injection were augmented or attenuated by previous doses in a day. At the time of study, lambs in this protocol had a similar postnatal age, duration of postthoracotomy recovery, and gender distribution as those in protocol 1 .

Twelve lambs in group 1 had sequential injections of $1.0 \times$ $10^{9} \mathrm{cfu}$ of heat-killed GBS ranging between one and four doses in a single day ( $n=24$ total doses); four lambs in group 1 and four lambs in group 2 each had four sequential i.v. injections of $1.0 \times 10^{9}$ cfu heat-killed GBS in a single day $(n=16$ doses in each group). Thus, 56 total $1.0 \times 10^{9}$-cfu doses were given. The $1.0 \times 10^{9}$-cfu dose was chosen because pilot data had indicated that it would elevate mean $\mathrm{P}_{\mathrm{pa}}$ by approximately $100 \%$ in the average-sized lamb weighing $4-5 \mathrm{~kg}$. At least $30 \mathrm{~min}$ elapsed between doses, and hemodynamic responses were permitted to stabilize before subsequent doses as in protocol 1. Hemodynamic measurements and arterial blood gas samples were obtained in the same time sequences as in protocol 1 .

Statistical Analysis. All data analysis was performed using the microcomputer version of Statistical Analysis Systems (SAS, SAS Institute, Cary, NC).

The stepwise multiple regression technique (27) was used to develop models that would describe the variability of the response of six hemodynamic variables $\left(\mathrm{P}_{\mathrm{pa}}, \mathrm{R}_{\mathrm{p}}, \mathrm{P}_{\mathrm{sa}}, \mathrm{R}_{\mathrm{s}}, \mathrm{Q}_{\mathrm{pa}}\right.$, and $\left.\mathrm{HR}\right)$ to injections of GBS. Responses considered in the models included the absolute change and the percentage of change of the response variable from baseline. Independent variables and their values considered in the models included dose of GBS (absolute and 
$\log$, corrected for weight of the lamb), the three treatment groups coded as two dichotomous variables, two interaction terms of dose and treatment groups, gender (GENDER: male $=1$, female $=2$ ), postnatal age in days (AGE), and duration of postoperative recovery in days (POD). The codes for the three treatment groups were as follows: the value for the variable GP1 $=1$ when the treatment was group 1. otherwise GP1 $=0$; the value for the variable GP2 = 1 when the treatment was group 2, otherwise $\mathrm{GP} 2=0$. As a result, when the treatment was group 3, GP1 and GP2 were 0 . Variables not reaching significance were then removed and the regression analysis was repeated to achieve the best model. Plots of the residual versus predicted values for all variables revealed random patterns and no important outliers were detected. A $p$ value $<0.05$ was considered a statistically significant relationship; the best-fit model was then described by the appropriate regression equation.

To detect any confounding change in other relevant variables, a two-factor ANOVA for repeated measures was used to test the response of mean atrial pressures, stroke volume, $\mathrm{PaO}_{2}, \mathrm{PaCO}_{2}$, $\mathrm{SaO}_{2}$, and pH. A similar one-factor ANOVA was used to detect whether changes in baseline values of the variables analyzed in the regression analysis occurred with the multiple injections throughout a day. A similar two-factor ANOV A evaluated the peak response of the multiple $10^{9}$-cfu injections in a day in protocol 2 to assess augmentation or attenuation of response with repeated doses. When an ANOVA indicated statistical significance at the $p<0.05$ level, follow-up group comparisons were performed with the Student-Newman-Keuls test. All variance was reported as SEM.

\section{RESULTS}

Protocol 1. In the analysis of each variable, the log of the GBS dose (log dose) and the percentage of change of the variable from the baseline value provided a better prediction of response than absolute dose and absolute change, respectively. Responses of the cardiovascular variables to injection of heat-killed GBS were immediate in onset, peak response typically occurred within 30 to $90 \mathrm{~s}$, and variables had returned to baseline values by $10 \mathrm{~min}$, as previously reported $(24,25)$.

Baseline values. Repeated baseline values for all hemodynamic and respiratory variables were not different either across the several injections performed in $1 \mathrm{~d}$ or between treatment groups (ANOVA, $p>0.05$ ); therefore, baseline values for these variables were cumulated for convenience. Baseline values for mean atrial pressures, stroke volume, $\mathrm{PaO}_{2}, \mathrm{PaCO}_{2}, \mathrm{pH}$, and $\mathrm{SaO}_{2}$ are found in Table 1. Baseline values for variables used in the regression analysis were (mean \pm SEM): $P_{p a} 16.6 \pm 0.5 \mathrm{~mm} \mathrm{Hg}, R_{p} 0.0580$ $\pm 0.0027 \mathrm{~mm} \mathrm{Hg} \cdot\left(\mathrm{mL} \cdot \mathrm{min}^{-1} \cdot \mathrm{kg}^{-1}\right)^{-1} \cdot \mathrm{P}_{\mathrm{sa}} 73.6 \pm 0.9 \mathrm{~mm} \mathrm{Hg}$.
$\mathrm{R}_{\mathrm{s}} 0.2712 \pm 0.0797 \mathrm{~mm} \mathrm{Hg} \cdot\left(\mathrm{mL} \cdot \mathrm{min}^{-1} \cdot \mathrm{kg}^{-1}\right)^{-1}, \mathrm{Q}_{\text {pa }} 294 \pm 9$ $\mathrm{mL} \cdot \mathrm{min}^{-1} \cdot \mathrm{kg}^{-1}$, and HR $206 \pm 4$ beats $\cdot \mathrm{min}^{-1}$

Pulmonary circulation. GBS injection of all three types caused a dose-dependent increase in mean $P_{p a}$ and $R_{p}$ (Fig. 1). The increase in $\mathrm{P}_{\mathrm{pa}}$ was positively influenced not only by the dose of GBS but was additionally influenced by the type of GBS and by the duration of postoperative recovery; the type of GBS used in group 1 resulted in a greater increase in $\mathrm{P}_{\mathrm{pa}}$ than the types used in groups 2 and 3 . The regression equation was as follows: $\%$ change $P_{\mathrm{pa}}=123.8+91.2(\log$ dose $)+45.7(\mathrm{GP} 1)+3.1(\mathrm{POD})$ $(p=0.0001, r=0.80)$. Using this regression equation, a lamb 3 $\mathrm{d}$ postoperative given a GBS dose of $1.5 \mathrm{~mL} / \mathrm{kg}$ would be predicted to have a peak $\mathrm{P}_{\mathrm{pa}}$ response to the different types of GBS as follows: group $1 \%$ increase $=123.8+91.2(0.176)+$ $45.7(1)+3.1(3)=195 \%$; groups 2 and $3 \%$ increase $=123.8$ $+91.2(0.176)+45.7(0)+3.1(3)=149 \%$.

The response of $R_{p}$ was also influenced by the dose of GBS, the type of GBS, and the interaction term of dose of group 2 (Fig. 1): the types of GBS used in groups 1 and 2 resulted in a greater increase in $R_{p}$ than the type used in group 3 . The regression equation was as follows: $\%$ change $R_{p}=130.1+117.4$ $(\log$ dose $)+92.0(\mathrm{GP} 1)+133.5(\mathrm{GP} 2)+62.0(\log$ dose * GP2 $)$ $(p=0.000 \mathrm{i}, r=0.75)$.

Systemic circulation. Only factors related to the type of GBS used in group 1 produced a clear dose-dependent effect on the response of $\mathrm{P}_{\text {sa }}$ (Fig. 2); $\mathrm{P}_{\text {sa }}$ was not influenced by dose of all groups or by factors related to the other treatment groups. The regression equation for all injections was as follows: \% change $\mathrm{P}_{\mathrm{sa}}=6.4+25.1(\mathrm{GP} 1)+19.8\left(\log\right.$ dose $\left.^{*} \mathrm{GP} 1\right)(p=0.0001, r$ $=0.53$ ). Because only factors related to group 1 influenced the overall response of $\mathrm{P}_{\text {sa, }}$ indicating a qualitative difference in response to the three treatment groups, regression models were developed for the response to individual treatment groups. The regression equation for group 1 was as follows: \% change $\mathrm{P}_{\mathrm{sa}}=$ $31.5+19.8(\log$ dose $)(p=0.0001, r=0.55)$. Group 2 had no dose-dependent effect on $\mathrm{P}_{\mathrm{sa}}(p>0.05$, power $=0.99)$ and group 3 had a marginal effect that was not apparent in the overall model: \% change $\mathrm{P}_{\mathrm{sa}}=13.6+7.3(\log$ dose $)(p=0.05, r=0.48)$.

The response of $R_{5}$ was positively influenced by the dose of GBS and the type of GBS; the GBS used in group 1 resulted in a greater increase in $R_{\mathrm{s}}$ than the types used in groups 2 and 3 (Fig. 2). The regression equation was as follows: \% change $R_{\mathrm{s}}=$ $78.3+28.8(\log$ dose $)+32.7(\mathrm{GP} 1)(p=0.0002, r=0.41)$.

$Q_{p a}$ and $H R$. The response of $\mathrm{Q}_{\mathrm{pa}}$ to GBS injection was negatively influenced by the dose of GBS (Fig. 3) and duration of postoperative recovery and positively influenced by postnatal age of the lamb; that is, longer duration of recovery enhanced the depression of $\mathrm{Q}_{\mathrm{pa}}$ and advanced postnatal age protected against this depression. The equation was as follows: \% change

Table 1. Respiratory gas exchange, atrial pressures, and stroke volume data before and after injection of heat-killed GBS in conscious lambs*

\begin{tabular}{|c|c|c|c|c|c|c|c|}
\hline \multirow[b]{2}{*}{ Variable/time } & \multirow[b]{2}{*}{$\begin{array}{c}\text { Baseline }{ }^{\dagger} \\
(n=80)\end{array}$} & \multicolumn{6}{|c|}{ GBS dose $(\mathrm{mL}) \ddagger$} \\
\hline & & $\begin{array}{c}0.1 \\
(n=12)\end{array}$ & $\begin{array}{c}0.25 \\
(n=13)\end{array}$ & $\begin{array}{c}0.50 \\
(n=13)\end{array}$ & $\begin{array}{c}1.0 \\
(n=20)\end{array}$ & $\begin{array}{c}3.0 \\
(n=12)\end{array}$ & $\begin{array}{c}6.0 \\
(n=10)\end{array}$ \\
\hline $\mathrm{PaO}_{2}(\mathrm{kPa})$ & $11.2(0.2)$ & $11.5 \quad(0.4)$ & $11.1 \quad(0.3)$ & $11.5 \quad(0.3)$ & $10.7(0.4)$ & $10.1 \quad(0.3)$ & $10.7 \quad(0.3)$ \\
\hline $\mathrm{SaO}_{2}(\%)$ & $95 \quad(0.5)$ & $95 \quad(1.2)$ & $95 \quad(0.9)$ & $96 \quad(1.0)$ & $95 \quad(1.0)$ & $95 \quad(1.0)$ & $94 \quad(0.9)$ \\
\hline $\mathrm{PaCO}_{2}(\mathrm{kPa})$ & $5.5 \quad(0.1)$ & $5.3(0.2)$ & $5.3 \quad(0.2)$ & $5.6 \quad(0.2)$ & $5.7 \quad(0.2)$ & $5.6 \quad(0.2)$ & $5.7 \quad(0.2)$ \\
\hline $\mathrm{pH}$ & $7.39(0.01)$ & $7.39(0.01)$ & $7.39(0.01)$ & $7.38(0.01)$ & $7.38(0.01)$ & $7.39(0.02)$ & $7.40(0.01)$ \\
\hline $\mathrm{P}_{\mathrm{la}}(\mathrm{mm} \mathrm{Hg})$ & $0.5(0.2)$ & $0.7(0.4)$ & $0.5 \quad(0.6)$ & $0.8 \quad(0.4)$ & $2.2(0.5)$ & $1.3(0.7)$ & $2.0(1.0)$ \\
\hline$P_{\mathrm{ra}}(\mathrm{mm} \mathrm{Hg})$ & $-0.6 \quad(0.2)$ & $-0.6(0.5)$ & $-0.5 \quad(0.7)$ & $0.3 \quad(0.5)$ & $1.7(0.6)$ & $0.6 \quad(0.8)$ & $1.7 \quad(0.9)$ \\
\hline Stroke volume $\left(\mathrm{mL} \cdot \mathrm{kg}^{-1} \cdot\right.$ beat $\left.^{-1}\right)$ & $1.5(0.1)$ & $1.5(0.1)$ & $1.5(0.1)$ & $1.4(0.1)$ & $1.5(0.1)$ & $1.2(0.1)$ & $1.4 \quad(0.1)$ \\
\hline
\end{tabular}

* Data are mean $(\mathrm{SEM}): 1 \mathrm{kPa}=0.1333 \mathrm{~mm} \mathrm{Hg} ; n=80$ total injections. $\mathrm{P}_{\mathrm{la}}$, mean left atrial pressure; $\mathrm{P}_{\mathrm{ra}}$. mean right atrial pressure. No differences between baseline and posttreatment values were observed for any variable (ANOVA, $p>0.05$ ).

+ All preinjection baseline and postinjection dose-group values were cumulated because no differences were detected across dose or treatment groups (ANOVA, $p>0.05$ ).

$\ddagger$ Refers to values $5 \mathrm{~min}$ after (for $\mathrm{pH}$ and gas tensions) and peak response (for $\mathrm{P}_{\mathrm{la}}, \mathrm{P}_{\mathrm{ra}}$, and stroke volume) after each dose of heat-killed GBS $\left(10^{9} \mathrm{cfu} \cdot \mathrm{mL}^{-1}\right)$. 

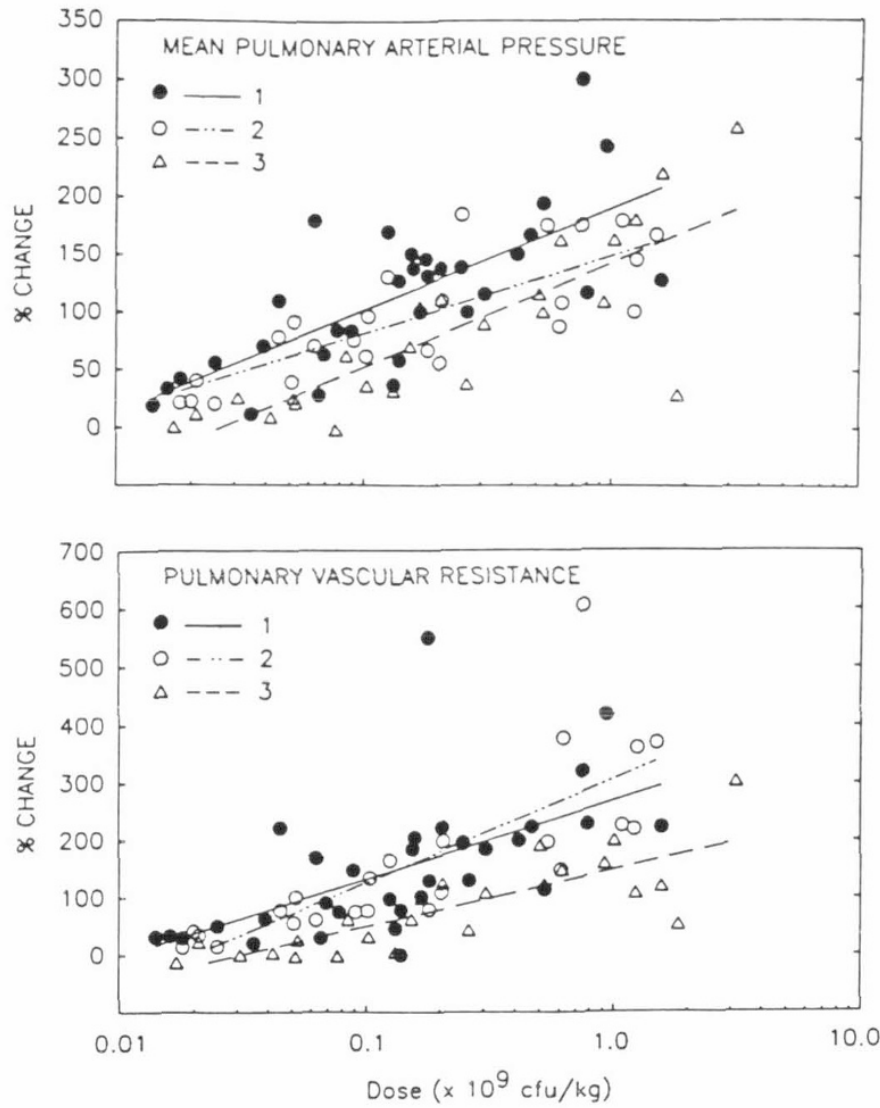

Fig. 1. Pulmonary vascular response of conscious lambs to three strains of heat-killed GBS, dose range $0.1-6.0 \times 10^{9} \mathrm{cfu} \cdot \mathrm{mL}^{-1}$, i.v. $n=$ 32 injections in treatment group 1 (type Ib), 24 injections in group 2 (type Ib), and 24 injections in group 3 (type III). Numbers in inset represent treatment groups; $y$ axis represents \% change from baseline. GBS injection of all three strains caused dose-dependent increases in $P_{p a}$ and $\mathrm{R}_{\mathrm{p}}$; group 1 bacteria had a more potent effect on $\mathrm{P}_{\mathrm{pa}}$, whereas groups 1 and 2 had additional influence on $R_{p}$.

$\mathrm{Q}_{\mathrm{pa}}=-32.4-13.6(\log$ dose $)-2.4(\mathrm{POD})+2.3(\mathrm{AGE})(p=$ $0.0001, r=0.57$ ). The response of HR was influenced negatively by the dose of GBS (Fig. 3) and positively by age and gender; that is, advanced postnatal age and female gender protected against the depression of $\mathrm{HR}$. The regression equation was as follows: $\%$ change $\mathrm{HR}=-42.3-10.1$ (log dose $)+5.5(\mathrm{GEN}-$ $\mathrm{DER})+0.6(\mathrm{AGE})(p=0.0001, r=0.58)$.

Atrial pressures and stroke volume. Mean atrial pressures and stroke volume did not differ between preinjection baseline and the postinjection peak response over the range of doses studied and responses did not differ between treatment groups; therefore, values for these variables were cumulated (ANOVA, all $p>0.05$, Table 1).

Arterial blood gas tensions, $\mathrm{pH}, \mathrm{SaO}_{2} . \mathrm{PaO}_{2}, \mathrm{SaO}_{2}, \mathrm{PaCO}_{2}$, and $\mathrm{pH}$ did not differ between preinjection baseline and the 5 -min postinjection periods over the range of doses studied and responses did not differ between treatment groups; therefore, values for these variables were cumulated (ANOVA, all $p>0.05$, Table $1)$.

Initial depressor response. Although the most important response to injection of heat-killed GBS in these studies was a pressor response of both pulmonary and systemic circulations, an initial depressor response that was immediate, brief, and small in magnitude was commonly observed.

A pulmonary depressor response was observed in 20 of the 80 total injections $(25 \%)$ and ranged in magnitude from -1 to -6 $\mathrm{mm} \mathrm{Hg}$ (mean $-2.4 \mathrm{~mm} \mathrm{Hg}$ ) decrease from baseline; a systemic depressor response was observed in 14 of the 80 injections $(18 \%)$ and ranged in magnitude from -3 to $-15 \mathrm{~mm} \mathrm{Hg}$ (mean -8.1
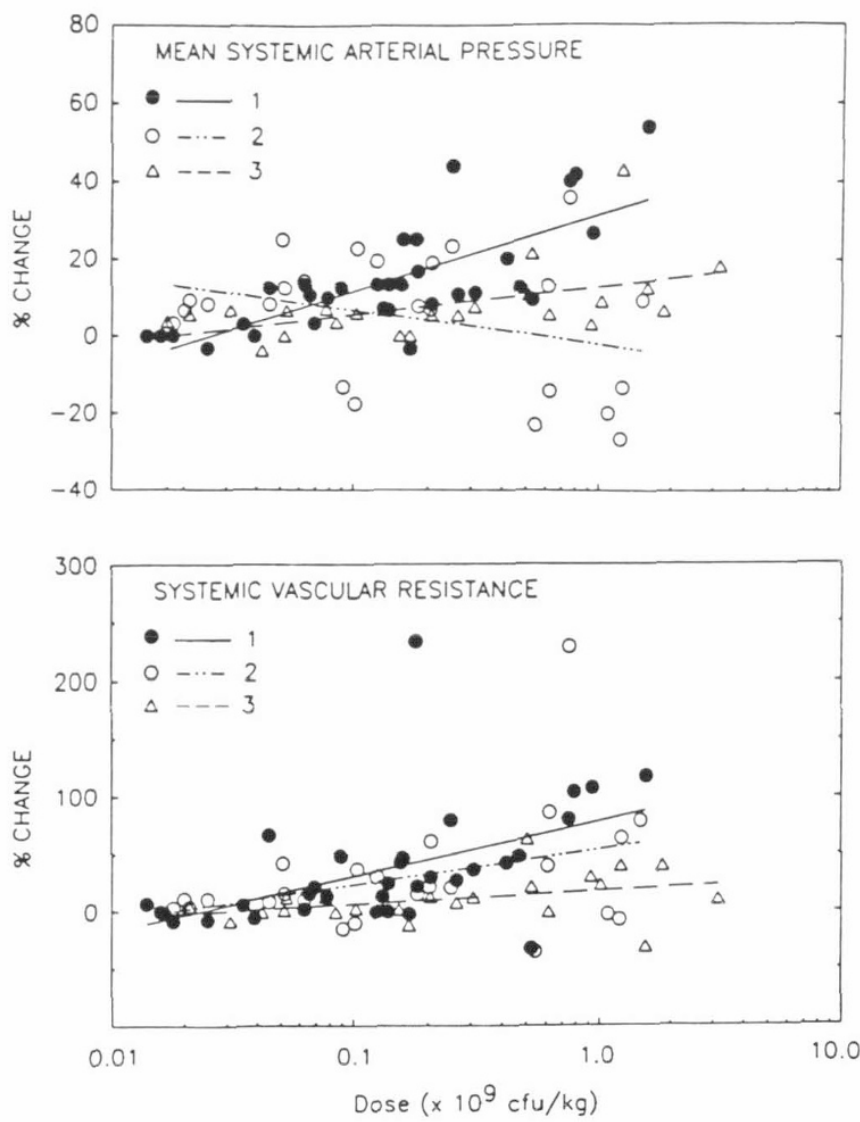

Fig. 2. Systemic vascular response of conscious lambs to three strains of heat-killed GBS, dose range $0.1-6.0 \times 10^{9} \mathrm{cfu} \cdot \mathrm{mL}^{-1}$, i.v. $n=32$ injections in treatment group 1 (type Ib), 24 injections in group 2 (type Ib), and 24 injections in group 3 (type III). Numbers in inset represent treatment groups; $y$ axis represents \% change from baseline. GBS injection of only the strain used in group 1 caused a dose-dependent increase in $\mathrm{P}_{\mathrm{sa}}$; GBS injection of all three strains caused a dose-dependent increase in $\mathrm{R}_{\mathrm{s}}$; group 1 bacteria had a more potent effect on $\mathrm{R}_{\mathrm{s}}$ than bacteria used in groups 2 and 3 .

$\mathrm{mm} \mathrm{Hg}$ ). The duration until recovery of this response was less than $30 \mathrm{~s}$. The depressor responses observed in the pulmonary and systemic circulations commonly occurred together; that is, the incidence of the systemic depressor response was greater when a similar pulmonary response also occurred compared with no such pulmonary response (40 versus $10 \%, \chi^{2}=9.4, p=$ $0.002)$.

The observation of the depressor responses was also strongly associated with the treatment group. A pulmonary depressor response was observed in $31 \%$ of injections in group $1,42 \%$ in group 2, and was not observed at all in group $3\left(\chi^{2}=15.3, p<\right.$ 0.0001 ). Similarly, a systemic depressor response was observed in $13 \%$ of injections in group $1,42 \%$ in group 2 , and was not observed at all in group $3\left(\chi^{2}=12.2, p<0.002\right)$.

We used the same multiple regression techniques and variables that evaluated the pressor responses to generate models to best describe the magnitude of pulmonary and systemic depressor responses. The models demonstrated that the absolute magnitude of the pulmonary pressure drop was related to duration of postoperative recovery and the magnitude of the systemic pressure drop was related to gender; that is, less magnitude of a $\mathrm{P}_{\mathrm{pa}}$ depressor response was observed with longer duration of postthoracotomy recovery, whereas less $\mathrm{P}_{\mathrm{sa}}$ response was observed with female gender. The magnitude of the depressor responses were not related to dose of GBS or postnatal age, were not different between treatment groups 1 and 2, and were not associated with decreases in atrial filling pressures or $\mathrm{Q}_{\mathrm{pa}}$; in fact, $\mathrm{Q}_{\mathrm{pa}}$ commonly increased with this response. 

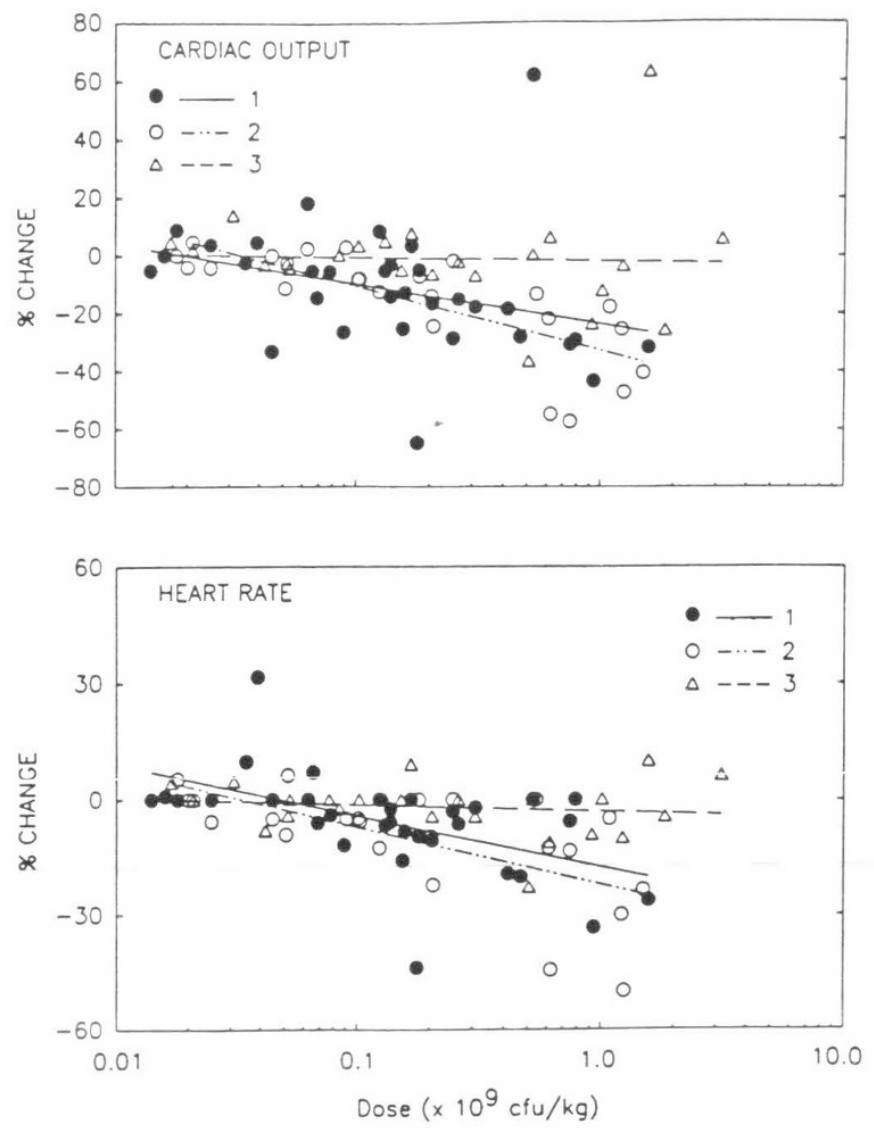

Fig. 3. $Q_{p a}$ and $H R$ responses in conscious lambs to three strains of heat-killed GBS, dose range $0.1-6.0 \times 10^{9} \mathrm{cfu} \cdot \mathrm{mL}^{-1}$, i.v. $n=32$ injections in treatment group 1 (type Ib), 24 injections in group 2 (type Ib), and 24 injections in group 3 (type III). Numbers in inset represent treatment groups; $y$ axis represents \% change from baseline. GBS injection of all three strains caused dose-dependent decreases in $\mathrm{Q}_{\mathrm{pa}}$ and $\mathrm{HR}$ without a treatment difference between the three strains.

Protocol 2. Up to four sequential $10^{9}$-cfu doses of heat-killed GBS did not produce attenuation or augmentation of cardiovascular responses. $\mathrm{P}_{\mathrm{pa}}$ increased a mean $18 \pm 1 \mathrm{~mm} \mathrm{Hg}$ over baseline in response to all $10^{9}$-cfu injections ( $122 \%$ mean increase for group 1, 103\% for group 2, and $93 \%$ for group 3) without a difference between sequential injections (ANOVA, $p>0.05$ ). Similarly, $\mathrm{P}_{\mathrm{sa}}$ increased a mean $8 \pm 1 \mathrm{~mm} \mathrm{Hg}(10 \%)$ in response to all sequential injections (mean $10 \pm 2 \mathrm{~mm} \mathrm{Hg}, 13 \%$ increase for group 1) without a difference between injections (ANOVA, $p>0.05)$. $\mathrm{Q}_{\mathrm{pa}}\left(\right.$ mean $\left.-17 \pm 6 \mathrm{~mL} \cdot \mathrm{min}^{-1} \cdot \mathrm{kg}^{-1},-7 \%\right)$ and $\mathrm{HR}$ (mean $-10 \pm 3$ beats $\cdot \min ^{-1},-5 \%$ ) both decreased from baseline. although negligibly, in response to all sequential $10^{9}$-cfu injections without a difference between dose number (ANOVA, $p>$ 0.05 ). No differences in response to sequential injections were found among treatment subgroups (ANOVA, $p>0.05$ ). The magnitude of responses of each variable was influenced by the treatment group as described in the results of protocol 1 . As in protocol 1, $\mathrm{PaO}_{2}, \mathrm{SaO}_{2}, \mathrm{PaCO}_{2}, \mathrm{pH}$, atrial pressures, and stroke volume did not differ between preinjection baseline and the postinjection periods.

Recovery. With one exception, all lambs recovered uneventfully without distress and thrived after these studies of serial injections of heat-killed GBS in a day. The exception was a single 8-d-old, 5.5-kg female lamb studied in treatment group 2 who went on to receive additional larger doses of GBS not evaluated in these protocols, including a total for the day of $24.9 \mathrm{~mL}(4.5$ $\left.\times 10^{9} \mathrm{cfu} \cdot \mathrm{kg}^{-1}\right)$ and a final dose of $9.0 \mathrm{~mL}\left(1.6 \times 10^{9} \mathrm{cfu} \cdot \mathrm{kg}^{-1}\right)$. This final high dose of heat-killed GBS caused a $21 \%$ decrease in $\mathrm{Q}_{\mathrm{pa}}$, which recovered within $5 \mathrm{~min}$. This lamb developed abdominal distension after enteral feeding and died several hours after the study; postmortem examination demonstrated distended bowel with necrotic areas.

\section{DISCUSSION}

Sepsis from group B beta-hemolytic streptococcus remains an important cause of neonatal morbidity and mortality. Previous studies of various serotypes of GBS isolated from infected human neonates and infused into animals have demonstrated that GBS causes important hemodynamic alterations. This study was conducted to further evaluate the influence of important experimental conditions on the hemodynamic response to GBS in our conscious lamb model. Specifically, the two main purposes were to examine whether GBS isolated from infected human neonates with different clinical syndromes and serotypes would cause different hemodynamic responses and whether other experimental factors may also influence these responses. We intentionally designed the study to evaluate the hemodynamic responses to doses of GBS that would not cause secondary and confounding changes in arterial $\mathrm{pH}$ and blood gas tensions, atrial pressures, or cardiac stroke volume and that would permit recovery of the chronically instrumented lamb.

The most reproducible hemodynamic effect of GBS is pulmonary hypertension. Pulmonary hypertension caused by GBS has been observed in human neonates $(5,7)$ and is a welldocumented response in several animal models to live GBS (8$16,19-22)$ or to heat-killed GBS $(23-25,28)$. In addition, we and others have shown that conscious animals may recover uneventfully from repeated injections of either heat-killed GBS (23-25) or type III GBS polysaccharide toxin $(17,18,29-32)$, an observation that provides a methodologic advantage to sequential experimental studies. In yearling sheep, Rojas et al. (14) have demonstrated that the pulmonary hypertensive response is similar between live GBS, heat-killed GBS, and the polysaccharide toxin; in neonatal lambs, we (24) also demonstrated that the pulmonary hypertensive response is similar between live GBS and heat-killed GBS. In this study, we have reconfirmed the dose-dependent pulmonary hypertensive effect of heat-killed GBS in conscious lambs and have provided regression models that predict hemodynamic response to GBS; in addition, this study establishes the importance of other factors on the magnitude of the pulmonary hypertensive response.

These data indicate that the quantitative degree of pulmonary hypertension may be influenced by the strain of GBS studied; specifically, GBS strains used in treatment groups 1 (serotype Ib, early-onset sepsis) and 2 (Ib, necrotizing enterocolitis) caused more pulmonary vasoconstriction than group 3 (III, meningitis). To our knowledge, this differential influence of individual GBS strains has not been previously reported. Furthermore, the duration of recovery from surgery in our chronically instrumented lambs also directly influenced the magnitude of pulmonary hypertensive response to heat-killed GBS. Although hemodynamic responses in chronically instrumented lambs are generally considered to be influenced by duration of postoperative recovery (33), we have now quantified the magnitude of this influence for studies of heat-killed GBS in conscious lambs.

This study also demonstrates a qualitative, treatment groupdependent difference in the response of $\mathrm{P}_{\mathrm{sa}}$ to GBS that has not been previously observed. Lambs receiving the GBS strain used in group 1 had a clear dose-dependent systemic pressor response to heat-killed GBS, whereas those receiving the GBS used in group 3 had a marginal effect; the GBS strain used in group 2 had no effect on $\mathrm{P}_{\mathrm{sa}}$. $\mathrm{R}_{\mathrm{s}}$ was positively influenced by dose of GBS; once again, the strain used in group 1 produced more potent systemic vasoconstriction. $\mathrm{Q}_{\mathrm{pa}}$ and $\mathrm{HR}$ were depressed by GBS in a dose-dependent manner and not influenced by treatment group. However, the dose-dependent depression of $\mathrm{Q}_{\mathrm{pa}}$ produced by heat-killed GBS was protected by advanced postnatal age and accentuated by longer duration of postoperative 
recovery; the apparent discrepant influences of advanced postnatal age and longer duration of postoperative recovery on $\mathrm{Q}_{\mathrm{pa}}$ remain unexplained by these data. Similarly, the dose-dependent depression of HR produced by heat-killed GBS was protected by advanced postnatal age and female gender; to our knowledge, this observation and the female gender protection against the initial pulmonary depressor effect of heat-killed GBS are unique observations that gender may be important to cardiovascular responses in the neonatal period.

The third purpose of this study was to determine whether repetitive doses of heat-killed GBS in a single day would attenuate or augment subsequent hemodynamic responses to GBS. This study demonstrated that sequential doses of 0.2 to $0.3 \mathrm{~mL} \cdot \mathrm{kg}^{-1}$ $\left(10^{9} \mathrm{cfu} \cdot \mathrm{mL}^{-1}\right)$ of heat-killed GBS given i.v. to neonatal lambs in a single day could produce a potent and reproducible elevation of $\mathrm{P}_{\mathrm{pa}}$ (mean $108 \%$ increase for all three groups) without attenuation or augmentation of response. This dose also provided negligible influence on other systemic variables and is an optimal dose in this model for primarily studying isolated pulmonary vascular effects of GBS. This observation of no attenuation or augmentation to serial doses is in contrast to the study of Philips et al. (23), in which a 2.5 -fold potentiation of the pulmonary pressor response to heat-killed GBS in conscious piglets was observed with single daily doses of $1 \times 10^{8} \mathrm{cfu}(10$-fold less than doses evaluated in this study) over 2 wk. Hemming et al. (34), using a trichloracetic acid extract of GBS, demonstrated that rechallenge $48 \mathrm{~h}$ after a first dose resulted in a 2 -fold augmentation of the pulmonary pressure response in oophorectomized adult ewes. The failure to observe attenuation or augmentation of hemodynamic responses in a single day in our study provides the methodologic advantage of studying cardiovascular responses before and after experimental interventions in the same day ( 24 . 25).

The initial depressor response of the pulmonary and systemic circulations to heat-killed GBS was an intriguing observation. This response was immediate, brief, isolated to treatment groups 1 and 2, and, although interrelated between the two circulations, was not observed with every dose. Interestingly, regression analysis indicated that the magnitude of this response was influenced by duration of postoperative recovery for $\mathrm{P}_{\mathrm{pa}}$ and by gender for $\mathrm{P}_{\text {sa }}$; that is, a smaller depressor response was observed for longer duration of postoperative recovery and female gender for these two variables, respectively. This response was not related to changes in atrial filling pressures or to a decrease in $\mathrm{Q}_{\mathrm{pa}}$; in fact, $\mathrm{Q}_{\mathrm{pa}}$ typically increased with this response. To our knowledge, this depressor response and its related factors are unique observations with GBS injection.

However, a similar initial depressor response has been reported to be typical of the systemic but not pulmonary vascular response to leukotriene injection. Ahmed et al. (35) observed a similar initial systemic depressor response to injected leukotriene $\mathrm{D}_{4}$ before an ultimate systemic pressor response in conscious adult sheep; indomethacin blocked the depressor response, leading the authors to conclude that this response was cyclooxygenase-mediated. A similar biphasic response of $R_{5}$ to leukotriene $D_{4}$ injection in neonatal lambs was observed by both Yokochi et al. (36) and, inconsistently, by Schreiber et al. (37). In spontaneously hypertensive rats, very high dose leukotriene $\mathrm{D}_{4}$ injection (20 $\mu \mathrm{g} / \mathrm{kg}$ intraarterially) has produced a similar systemic vasodepressor effect followed by a pressor response; however, this initial depressor effect was not observed in normotensive rats (38).

The mechanism of the dissimilar cardiovascular responses to different strains of heat-killed GBS was not investigated in this study; however, existing information suggests that organismrelated factors are, in part, responsible. O'Brien et al. (39) and Hemming et al. (34) demonstrated that a trichloroacetic acid extract of type III GBS produces pulmonary hypertension in both lambs (39) and adult oophorectomized ewes (34); these authors observed qualitatively different $\mathrm{P}_{\mathrm{sa}}$ responses in these two different age groups that were unexplained. Before this,
Hellerqvist et al. $(31,32)$ extensively fractionated type III GBS and isolated and studied a pure polysaccharide toxin capable of causing pulmonary hypertension when injected into yearling sheep; this toxin is different from either group B or type III polysaccharides.

More recently, Gibson et al. (40) studied the pulmonary vascular response to live isogenic mutants of type III GBS in neonatal piglets; these investigators demonstrated that GBS mutants devoid of either type III capsular polysaccharide or $\beta$ hemolysin remained capable of producing pulmonary hypertension equivalent to the parent type III GBS strain. These data led these authors to conclude that type III polysaccharide or $\beta$ hemolysin were not essential for type III GBS to induce the acute effects of GBS sepsis, including pulmonary hypertension and impaired respiratory gas exchange. Finally, Gibson et al. (41) have also reported that two additional gram-positive species (Enterococcus and Staphylococcus epidermidis) produce pulmonary hypertension and elevation of circulating thromboxane B equivalent to those produced by live type III GBS in piglets; these data led the authors to suggest that components responsible for the hemodynamic effects of gram-positive bacteria in their model may be universally common or that the final common pathway may be stimulated by a number of nonspecific components. Whether a variable expression, concentration, or stimulatory potency of the physiologically active toxin or toxins occurs with various strains of GBS remains an unstudied possibility.

Finally, chronically instrumented lambs, with one exception, recovered uneventfully from these studies, providing a methodologic advantage to serial or paired studies, particularly in terms of studying responses before and after experimental interventions $(24,25)$. The finding that the sirgle lamb that died developed an ischemic gastrointestinal lesion after an unusually large load of the GBS isolated from the blood of an infant with necrotizing enterocolitis is another intriguing observation that suggests the possibility of a strain-dependent gastrointestinal or mesenteric vascular virulence.

In summary, we have confirmed the pulmonary and systemic pressor effects of heat-killed GBS in lambs; in addition, we have demonstrated strain-dependent quantitative differences in pulmonary vascular response and qualitative differences in systemic vascular response, as well as several important experimental factors that may influence hemodynamic responses to GBS. These factors should be accounted for in studies investigating cardiovascular responses to GBS.

Acknowledgments. The authors thank Dr. Kenneth Boyer for analyzing GBS serotypes and providing the GBS used in group 3, which was originally kindly provided to Dr. Boyer by Dr. Carol J. Baker of Baylor College of Medicine in Houston; Dr. William Meadow for providing the GBS used in group 1 and for assistance in GBS preparation; Lorna J. Torgerson, B.Sc., for providing vital technical assistance; and Colleen Garcia for preparing the manuscript.

\section{REFERENCES}

1. Howard JB. McCracken GH 1974 The spectrum of group B streptococcal infections in infancy. Am J Dis Child 128:815-818

2. Vollman JH, Smith WL, Ballard ET, Light IJ 1976 Early onset group B streptococcal disease: clinical, roentgenographic, and pathologic features. J Pediatr 89:199-203

3. Menke JA, Giacoia GP. Jockin H 1979 Group B beta hemolytic streptococcal sepsis and the idiopathic respiratory distress syndrome: a comparison. J Pediatr 94:467-47

4. Pass MA, Gray BM, Khare S, Dillon HC 1979 Prospective studies of group B streptococcal infections in infants. J Pediatr 95:437-443

5. Shankaran S, Farooki ZQ. Desai R $1982 \beta$-Hemolvtic streptococcal infection appearing as persistent fetal circulation. Am J Dis Child 136:725-727

6. Dillon HC, Khare S. Gray BM 1987 Group B streptococcal carriage and disease: a 6-year prospective study. J Pediatr 1 10:31-36

7. Hammerman C. Lass N, Strates E, Komar K. Bui K-C 1987 Prostanoids in neonates with persistent pulmonary hypertension. J Pediatr 110:470-472 
8. Runkle B. Goldberg RV. Streitfeld MM. Clark MR. Buron E. Setzer ES. Bancalari E 1984 Cardiovascular changes in group B streptococcal sepsis in the piglet: response to indomethacin and relationship to prostacyclin and thromboxane $\mathrm{A}_{2}$. Pediatr Res 18:874-878

9. Goldberg RN, Suguihara C. Streitfeld MM. Bancalari A. Clark MR. Bancalari E 1986 Effects of a leukotriene antagonist on the early hemodynamic manifestations of group B streptococcal sepsis in piglets. Pediatr Res 20:1004-1008

10. Meadow WL. Rudinsky BF. Strates E. Komar KJ 1987 Oxygen delivery. oxygen consumption. and metabolic acidosis during group B streptococcal sepsis in piglets. Pediatr Res 22:509-512

11. Meadow WL. Meus P. Rudinsky BF. Strates E 1986 Swine in Biomedical Research. Plenum Publishing Corp, New York, pp 461-471

12. Meadow WL. Meus PJ 1986 Early and late consequences of group B beta streptococcal sepsis in piglets: effects on systemic. pulmonary. and mesenteric circulations. Circ Shock 19:347-356

13. Meadow WL. Meus PJ 1985 Unsuspected mesenteric hypoperfusion despite apparent hemodynamic recovery in the early phase of septic shock in piglets. Circ Shock 15:123-129

14. Rojas J. Green RS. Hellerqvist CG. Olegard R. Brigham KL. Stahlman MT 1981 Studies on group B $\beta$-hemolytic streptococcus. II. Effects on pulmonary hemodynamics and vascular permeability in unanesthetized sheep. Pediatr Res 15:899-904

15. Truog WE. Sorensen GK. Standaert TA. Redding GJ 1986 Effects of the thromboxane synthetase inhibitor. Dazmegrel (UK 38.485). on pulmonary gas exchange and hemodynamics in neonatal sepsis. Pediatr Res 20:481-486

16. Huddleston KW. Lyrene RK. Dew A. Gray BM. Philips JB 1986 Influence of prostaglandin $\mathrm{D}_{2}$ on hemodynamic effects of group $\mathrm{B}$ streptococcus in neonatal lambs. Dev Pharmacol Ther 9:260-265

17. Engelhardt B. Sandberg K. Bratton D, van den Abbeele A. Grogaard J Hellerqvist C. Sundell H 1987 The role of granulocytes in the pulmonary response to group B streptococcal toxin in young lambs. Pediatr Res 21:159165

18. Sandberg K. Engelhardt B. Hellerqvist C. Sundell H 1987 Pulmonary response to group B streptococcal toxin in young lambs. J Appl Physiol 63:20242030

19. Truog WE. Gibson RL. Juul SE. Henderson WR. Redding GJ 1988 Veonatal group B streptococcal sepsis: effects of late treatment with Dazmegrel. Pediatr Res 23:352-356

20. Hammerman C. Komar K. Meadow W. Strates E 1988 Selective inhibition of thromboxane synthetase reduces group B beta-hemolytic streptococci-induced pulmonary hypertension in piglets. Dev Pharmacol Ther 11:306-312

21. Pinheiro JMB, Pitt BR. Gillis CN 1989 Roles of platelet-activating factor and thromboxane in group B streptococcus-induced pulmonary hypertension in piglets. Pediatr Res 26:420-424

22. Redding GJ. Gibson RL. Standaert TA. Truog WE 1990 Regional pulmonary blood flow in piglets during group B streptococcal bacteremia. Am Rev Respir Dis 141:1209-1213

23. Philips JB. Lyrene RK. Godoy G. Graybar G. Barefield E. Sams JEP. Gray BM 1988 Hemodynamic responses of chronically instrumented piglets to bolus injections of group B streptococci. Pediatr Res 23:81-85

24. Schreiber MD. Covert RF. Torgerson LJ 1992 Hemodvnamic effects of heat- killed group B $\beta$-hemolvtic streptococcus in newborn lambs: role of leukotriene $D_{4}$. Pediatr Res 31:121-126

25. Schreiber MD. Covert RF. Torgerson LJ 1992 Effect of aminophylline on the pulmonary and systemic hemodynamic response to group B beta-hemolytic streptococcus and leukotriene $\mathrm{D}_{4}$ in newborn lambs. J Dev Physiol 17:195200

26. Covert RF. Schreiber MD. Leff AR. White SR, Munoz NM. Torgerson LJ 1992 Oxvgen metabolism and catecholamine secretion during chloralose anesthesia in lambs. J Dev Physiol 17:125-132

27. Cody RP. Smith JK 1987 Applied Statistics and the SAS Programming Language. Elsevier Science Publishing Co. New York. pp 70-81

28. Tarpey MN. Graybar GB. Lyrene RK. Godoy G, Oliver J, Gray BM. Philips JB 1987 Thromboxane synthesis inhibition reverses group B streptococcusinduced pulmonary hypertension. Crit Care Med 15:644-647

29. Rojas J. Larsson LE, Ogletree ML. Brigham KL. Stahlman MT 1983 Effects of cyclooxygenase inhibition on the response to group B streptococcal toxin in sheep. Pediatr Res 17:107-110

30. Rojas J, Palme C, Ogletree ML. Hellerqvist CG, Brigham KL, Stahlman MT 1984 Effects of methylprednisolone on the response to group B streptococcal toxin in sheep. Pediatr Res 18:1141-1144

31. Hellerqvist CG. Rojas J. Green RS. Sell S. Sundell H. Stahlman MT 1981 Studies on group B 3 -hemolvtic streptococcus. I. Isolation and partial characterization of an extracellular toxin. Pediatr Res 15:892-898

32. Hellerqvist CG. Sundell H. Gettins P 1987 Molecular basis for group B $\beta$ hemolytic streptococcal disease. Proc Natl Acad Sci USA 84:51-55

33. Sidi D, Kuipers JRG. Heymann MA. Rudolph AM 1982 Recovery of cardiovascular function in newborn lambs after thoracotomy. Pediatr Res 16:705710

34. Hemming VG. OBrien WF. Fischer GW, Golden SM, Noble SF 1984 Studies of short-term pulmonary and peripheral vascular responses induced in oophorectomized sheep by the infusion of a group B streptococcal extract. Pediatr Res 18:266-269

35. Ahmed T. Marchette B. Wanner A. Yerger L 1985 Direct and indirect effects of leukotriene $\mathrm{D}_{4}$ on the pulmonary and systemic circulations. Am Rev Respir Dis 131:554-558

36. Yokochi K. Olley PM. Sideris E. Hamilton F. Huhtanen D. Coceani F 1982 Leukotrienes and other Lipoxygenase Products. Raven Press. New York. pp $211-214$

37. Schreiber MD. Heymann MA. Soifer SJ 1987 The differential effects of leukotriene $\mathrm{C}_{4}$ and $\mathrm{D}_{4}$ on the pulmonary and systemic circulations in newborn lambs. Pediatr Res 21:176-182

38. Feurstein G. Zukowska-Grojec Z. Kopin IJ 1981 Cardiovascular effects of leukotriene $\mathrm{D}_{4}$ in SHR and WKY rats. Eur J Pharmacol 76:107-110

39. O'Brien WF, Golden SM, Bibro MC. Charkobardi PK. Davis SE. Hemming VG 1985 Short-term responses in neonatal lambs after infusion of group B streptococcal extract. Obstet Gynecol 65:802-806

40. Gibson RL. Redding GJ. Truog WE. Henderson WR. Rubens CE 1989 Isogenic group B streptococci devoid of capsular polysaccharide or $\beta$-hemolysin: pulmonary hemodynamic and gas exchange effects during bacteremia in piglets. Pediatr Res 26:241-245

41. Gibson RL. Truog WE. Redding GJ 1988 Thromboxane-associated pulmonary hypertension during three types of gram-positive bacteremia in piglets. Pediatr Res 23:553-556 